ICHEU 2021

International Conference «Humanity in the Era of Uncertainty»

\title{
ECONOMIC AND PSYCHOLOGICAL FEATURES OF RUSSIANS' ATTITUDE TO MONEY AMID CORONAVIRUS PANDEMIC
}

\author{
Anatoly Laktionovich Zhuravlev (a)*, Juliet Albertovna Kitova (b) \\ *Corresponding author \\ (a) Institute of Psychology RAS, 129366 st. Yaroslavskaya, 13, building 1, Moscow, Russia, \\ alzhuravlev2018@yandex.ru \\ (b) Institute of Psychology RAS, 129366 st. Yaroslavskaya, 13, building 1, Moscow, Russia, \\ j-kitova@yandex.ru
}

\begin{abstract}
The analysis of a person's attitude to modern social phenomena is of high relevance in humanitarian studies. Personal attitudes are amenable to study through the analysis of the national environment and worldview attitudes, which are considered as unique, self-organizing in a hierarchical system of positions. Their content, structure, time perspective and hierarchical subordination of elements are subject to psychological analysis. An appeal to the information potential of the Internet enables to study the attitude of large nonformalized social groups to various phenomena taking into account regional, national or global trends. In this respect, the peculiarities of the population's attitude to money as economic resource are of considerable interest. Studies carried out among Russians have shown that their main expectations for improving living standards are associated with the state being the most stable economic entity amid uncertainty caused by the coronavirus pandemic. The greatest interest of users is attracted by income and expenses, while savings and investments do not cause proper understanding. Users' interest in entrepreneurial activity is so low that it can be compared with the rare interest in criminal sources of income. The consumer attitude to money can be defined as the main psychological background of the attitude of users of the social network Twitter to them. It would be important to consider the psychological characteristics of attitudes towards money among the representatives of other cultures (Japanese, Americans, etc.) for understanding the mechanisms of mutual influence of economic and psychological factors on the development of macroeconomic processes in society.
\end{abstract}

2357-1330 @ 2021 Published by European Publisher.

Keywords: Attitude to money, pandemic, content analysis, income, expense, savings 


\section{Introduction}

In modern domestic psychology, the study of a person's attitude to social phenomena is of high relevance. Personality relationships are formed in a certain national environment, express ideological attitudes, have a unique character, self-organize into a hierarchical system, thus enabling to study their content, structure, time perspective and level subordination of elements (Kitova, 2020; Kilbourne et al., 2005; Shvarts, 2008). In turn, an appeal to the Internet information potential enables to study the attitude to various phenomena of large non-formalized social groups regarding regional, national or global trends.

\section{Problem Statement}

The analysis of published works states that studies of the characteristic features of the attitude of the population to money as an economic resource (a factor in the economic development of a family or society) in Russian psychology have not yet been carried out.

\section{Research Questions}

The study of the attitude of the population to money, which is directly influenced by the sociocultural, situational and gender characteristics of the individual, value orientations and beliefs, its motivational characteristics, etc. can be singled out as an independent scientific field (Beaudreau, 2006; Chuah et al., 2009; Economic Psychology, 2000; Fenko, 2000; Polukhina, 2019). Money is considered as a factor of freedom and security, luck and failure, neuroses and internal conflicts, as well as a factor of maladaptive or irrational behaviour.

The state of the problems studied in this direction is most fully presented in the work of O.P. Polukhin "Psychology of money as an object of psychological research" (Polukhina, 2019, p. 27), and among the most recent works reference can be made to the research of Magera (2020) "Psychology of money amid social isolation" (p. 59). Both publications testify to the stable interest of specialists in the issue of money.

\section{Purpose of the Study}

The purpose of the study is to identify the structural and substantive features of the attitude towards money being an economic resource.

\section{Research Methods}

The object of the research is the messages of Russian users of the social network Twitter, in the texts of which the word money is present. 1787 messages were analyzed. The messages were collected by the method of continuous sampling throughout the territory of the Russian Federation from December 02, 2021 to December 03, 2021 during the coronavirus pandemic, which can certainly be considered as conditions of uncertainty. The study uses an automated word analysis system (Kitova, 2020). To assess the results 
obtained, quantitative (frequency analysis) and qualitative (content analysis) approaches are used, for the interpretation of research results, the methods of grounded theory and expert assessments are applied.

\section{Findings}

Results of the frequency analysis of the words found in user messages enabled to prepare a weighted (taking into account the frequency of use) list of 100 leading words (50 nouns, 50 verbs) used in messages about money. Words were studied as single categories (with no regard to the connections between them). The list of received words and their quantitative characteristics are presented in Figure 1, the frequency of use of each word correlates with the font size: the larger the font size, the higher the activity of using the presented word (Figure 1).

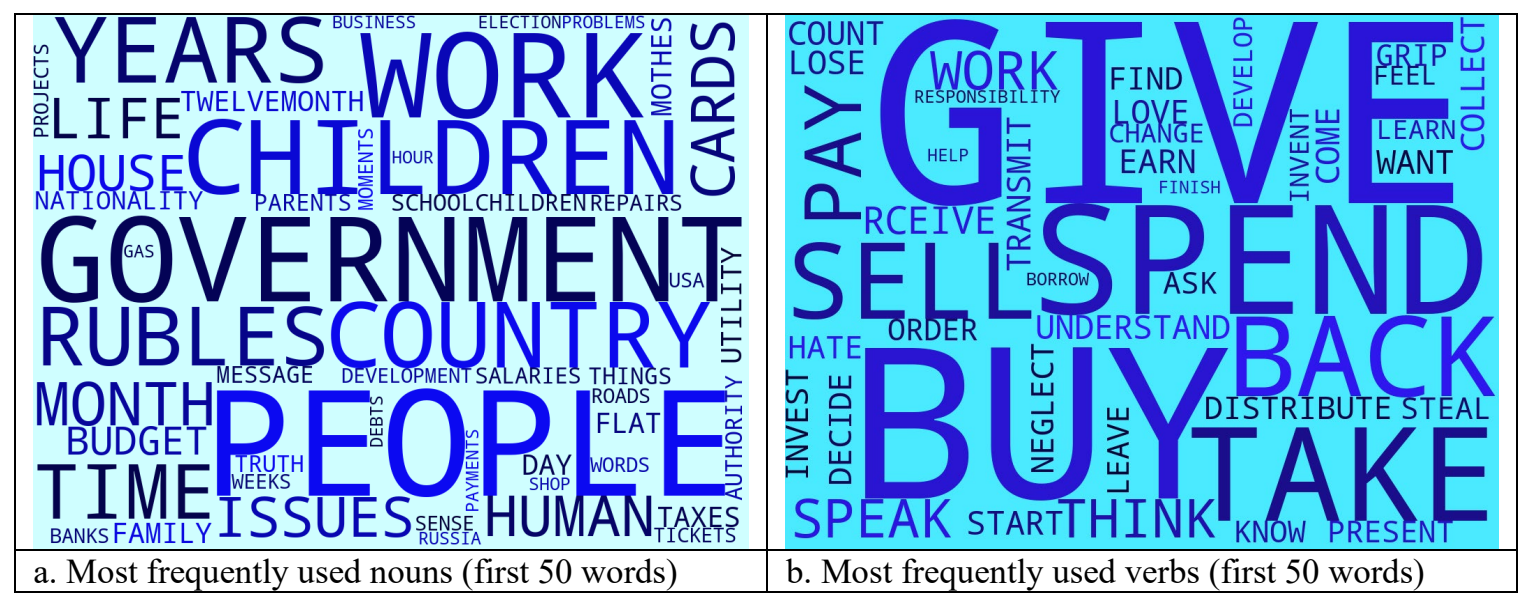

Figure 1. Frequency analysis of words in user messages containing the word "money"

The first three positions of the analyzed words with the highest repetition frequency were identified, each of which includes three words. Among the nouns highlighting the main objects and phenomena discussed in messages about money, the leading positions are occupied by three key words: "people (165) - government officials (137) - children (108)"; at the $2^{\text {nd }}$ level they are joined by the words "Russia (93) country (83) - years (79)"; on the $3^{\text {rd }}$ - "rubles (72) - month (67) - time (67)". Even with a cursory examination, it is obvious that money is most often considered in accordance with three leading categories being people (1), country (2) including government officials as representatives of the state and time (3). The latter category is reflected in words such as years, month, time. Thus, we can say that money is viewed by Russians primarily as a resource to meet the needs of people (and not the goals of economic activity), which can be defined as a consumer attitude towards them. Money is also associated with spending on children (it is obvious that we are talking about expenses since children do not replenish the family budget). The state is viewed by users as the main subject of ensuring the standard of living of Russians (up to 60\% of them work in budgetary organizations, pensioners and children are also supported by the state, which provides cash benefits, partial preservation of parental income and free education in general academic schools).

For further analysis of the resulting list of words, they were grouped according to semantic categories. The approach involves expert selection of specific features and the selection of the 
corresponding concepts (words). For example, the words people, person, children are combined under the general name "people" or, words such as years, month, week, minutes are classified as "time".

A comparison of the semantic meanings of words with their economic and non-economic connotations resulting in allocating material and non-material categories that reflect the attitude towards money were used as particular approaches. For example, frequency analysis showed the predominance of non-economic (humanitarian) words in the messages of users, such as people (165), children (108), human (63), life (61), family (51). Words from the category of the material group have lower indicators and they begin with the words thing (33) and continue (in decreasing order of importance) with words such as salary (30), repairs (30), debts (25), gas (24), etc. This approach once again notes the dominance of consumer orientation in the use of money aimed to meet the needs of children and families rather than at strengthening tangible assets and increasing them.

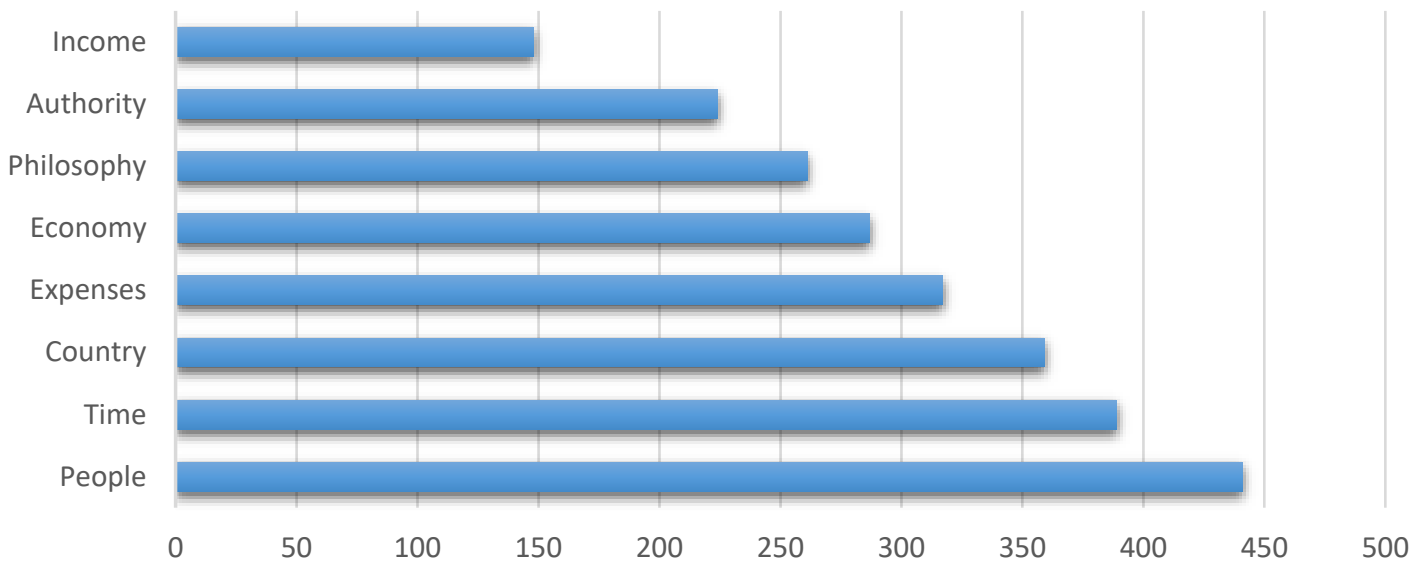

Figure 2. Structure of attitude to money by users of the social network Twitter.

Taking into account the described principles of analysis, Figure 2 shows the quantitative characteristics of messages regarding the frequency of mentioning already semantic categories (their simple number is taken into account) from the most discussed problems to the less mentioned positions (Figure 2). As expected, in the final list of semantic categories, money is primarily associated with the needs of people ( $1^{\text {st }}$ rank) and is represented by such words as home, children, family, etc. The second semantic category is assigned to the phenomenon of "time": users discuss the mutual influence of time and money, their ratio, irreversibility of time and the factor of its limitation/insufficiency (2). The $3^{\text {rd }}$ position is related to the discussion of the state's capabilities in ensuring and maintaining the income of the population (3). As a confirmation of this kind of expectation represented by the relationship between income and budget payments from the state, it is possible to point out the fact that the semantic category "income" (which is discussed in more detail below) in user messages is represented by only three categories of words: work, salary, payments.

At the $4^{\text {th }}$ level, the expenses (4) are discussed by users. The expenses are primarily related to the maintenance of children (as suggested above) and ensuring the needs of the family: the family and children are the main consumers of funds. A small number of messages contain words that reflect topics of leisure and communication, for example, cinema, theater, airplane or train tickets. Among the family's expenses, 
such items as taxes, utility bills, payments for gas consumption are also presented (when discussing the last item, negative comments are often heard). The $5^{\text {th }}$ most frequently used position is associated with the exchange of information about economic processes and subjects, in the messages of users such words as banks, rubles, accounts, taxes, business, etc. are used. This level is defined by us as economic processes and phenomena in society (5).

The $6^{\text {th }}$ position reflects the philosophical reasoning of users about the economic aspects of the life of a person and society (6). Specifically, the tasks of ensuring the financial well-being of the population, ways to achieve a certain level of quality of life are discussed, the standard of living in economically developed countries of the world is analyzed, questions about a fair/unfair order of the world and the methods of distribution of public goods are debated, etc.

The $7^{\text {th }}$ semantic category reflects different opinions in assessing the economic decisions of representatives of government bodies (7). More than anything else, they discuss the negative role of government officials in the regulation of socio-economic processes. The last position is occupied by reflections on possible sources and ways to increase the level of income (8).

Considerations on this matter, as noted above, are limited to three positions being work, earnings and payments. Reflections on the ways and methods of obtaining entrepreneurial income are almost never encountered. For example, the words "business" or "entrepreneurship" are not included in the top 50 words related to money, they are found in the final (low-frequency) part of the set of words in the analyzed messages. These data correlate with the results of sociological studies, which revealed a lack of experience in entrepreneurial activity among $97 \%$ of Russians (On pressing problems ..., 2019).

A meaningful analysis of the verbs discussed in the messages and carried out according to the above scheme enabled to single out the following semantic categories: expenses - spend, buy, pay; income receive, earn, get; social interaction, communication and exchange - help, present, borrow, give, give back; socio-economic activity (considered in the work without taking into account its expected economic effect) - do, start, order, make a deal; business - sell, income; emotions, i.e. attitude to money expressed in feelings - love, hate, want; intellectual features - know, think, understand; as well as talking about savings (keep, save), investments (invest) and criminal ways to increase income (steal, for example).

It is worth paying attention to the fact that thinking about the system of saving money is at a low level, as are the words representing business, i.e. it can be assumed that not many Russians have real experience of saving money.

In continuation, it is important to note that the level of interest in investments among users of social networks is comparable to the conviction of the criminal origin of big money and the inability to earn it in an honest way. 


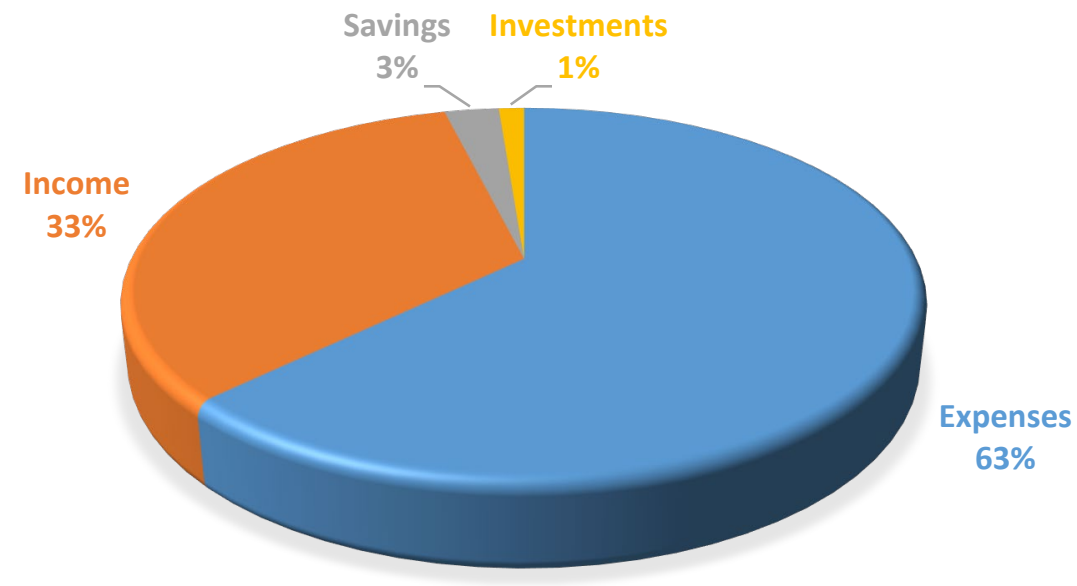

Figure 3. Four-factor structure of social network users' attitudes towards money

As it turned out, the structure of the attitude of users of the social network Twitter to money reveals bright features from an economic viewpoint (see: Figure 3). The first is related to the fact that the majority of users $(63 \%)$ are interested in consumption/spending money while the messages contain situations related to what they would like to buy, what to spend money on, or where to go to rest if they have money. Reflections on increasing incomes occupy the consciousness of users almost two times less (33\%), and the majority of the population is not active about savings and investments (1\%). This position once again shows the passive-consumer nature of the attitude towards money.

\section{Conclusion}

Generalization of the results obtained enables to draw several important conclusions:

- users associate the main expectations in improving the standard of living with the state being the most stable economic entity amid the coronavirus pandemic;

- if the economic structure of attitudes towards money is presented as a four-factor model, then the greatest interest of users is attracted by income and expenses, and savings and investments do not cause due attention;

-the interest of users in entrepreneurial activity (one of the leading resources for economic development) is so insignificant that it can be correlated in terms of the level of manifestation with a rare interest in criminal sources of income (less than $3 \%$ ).

- the predominance of consumer attitudes can be defined as the main psychological background of the attitude of users of the social network Twitter to money.

In the future, it is necessary to identify the prevailing emotional background of attitudes towards money, including the representatives of other cultures, for example, Japanese or Americans, which can have a certain impact on understanding the mechanisms of mutual influence of economic and psychological factors on macroeconomic processes in conditions of uncertainty and risks (Psychological Research, 2018; Zhuravlev \& Kitova, 2019). 


\section{Acknowledgments}

The study was carried out with the financial support of the Russian Science Foundation (RSF) within the framework of scientific project No. 21-18-00541.

\section{References}

Beaudreau, B. C. (2006). Identity, entropy and culture. Journal of Economic Psychology, 27(2), 205-223.

Chuah, S. H., Hoffmann, R., Jones, M., \& Williams, G. (2009). An economic anatomy of culture: Attitudes and behaviour in inter-and intra-national ultimatum game experiments. Journal of Economic Psychology, 30(5), 732-744.

Economic psychology. (2000). Sociocultural approach. Peter.

Fenko, A. B. (2000). The problem of money in foreign psychological research. Psychological Journal, $21(1), 50-62$.

Kilbourne, W., Grünhagen, M., \& Foley, J. (2005). A cross-cultural examination of the relationship between materialism and individual values. Journal of Economic Psychology, 26(5), 624-641.

Kitova, D. A. (2020). Microeconomic psychology of the subject of life. Publishing house "Institute of Psychology RAS".

Magera, T. N. (2020). Psychology of money in conditions of social isolation. Economy and entrepreneurship, 4(117) 59-61.

On the pressing problems of our life and the interaction of regulators, business and citizens / Report on the results of a mass sociological survey. (2019). https:/www.bankdelo.ru/news/100in1/pub/2570

Polukhina, O. P. (2019). Psychology of money as an object of psychological research. Finance of Russia in the context of globalization: materials of the IV International scientific and practical conference, timed to the "Day of the financier - 2019". Voronezh: Voronezh Institute of Economics and Law, 26-31.

Psychological research of global processes: preconditions, trends, prospects. (2018). Publishing house "Institute of Psychology RAS".

Shvarts, Sh. (2008). Cultural value orientations: the nature and consequences of national differences. Psychology. Journal of the Higher School of Economics, 5(2) 37-67.

Zhuravlev, A. L., \& Kitova, D. A. (2019). Socio-psychological factors of the economic development of Russian society in the context of digital technologies. Institute of Psychology of the Russian Academy of Sciences. Social and economic psychology, 4(16) 6-42. 